\title{
Ptosis Surgery with an Improved Method of Shortening the Müller's Muscle and the Levator Aponeurosis with Transconjunctival Threading
}

\author{
Kosuke Ogasawara* \\ Medical Corporation, Ogasawara Eye Clinic, Japan
}

\begin{abstract}
Purpose: To report an improved method of operative treatment for ptosis wherein shortening of the Müller's muscle and the levator aponeurosis could be performed with transconjunctival threading without making an incision in the tarsal muscle.

Methods: 1) After ophthalmic anesthesia administration, the tarsus was pinched so that the tip of fixation forceps was approximately $5 \mathrm{~mm}$ from the eyelid, and the tarsus was reversed. 2) A small dose of local anesthesia (1\% lidocaine) was administered at a site approximately $5 \mathrm{~mm}$ from the tip of the pinched forceps, and a high-frequency scalpel was used to make an incision approximately $5 \mathrm{~mm}$ wide in the conjunctiva. 3) A 7-0 nylon double-armed thread was passed through the Müller's muscle on the tarsal side (approximately $3 \mathrm{~mm}$ from the eyelid margin) under direct vision. Furthermore, from the tarsal side, the thread was passed through the intra-tarsal layer (approximately $5 \mathrm{~mm}$ from the insertion point of the needle) and subsequently, rethreading was performed through the incision point of the tarsus to the conjunctival fornix. 4) At that site, the thread was passed from a site superior to the Müller's muscle in a direction traversing the levator aponeurosis, and suturing was performed. 5) After confirming the eyelid elevation was good and symmetrical, bonding of the site of the conjunctival incision was performed with coagulation.

Results: The present procedure was performed in 17 patients with age-related ptosis. Postoperatively, satisfactory aesthetic results were achieved in all cases. No particular complications were observed, and there was no case of overcorrection or incomplete eyelid closure. Mean operative time was 11 minutes.

Conclusion: When the present procedure was used, the operative duration was shortened, patient burden was reduced, and expansion of the indication range of age-related ptosis could be achieved, suggesting that the present operative method may be a preferred method for initial surgery in age-related ptosis.
\end{abstract}

\section{Keywords}

Ptosis surgery, Transconjunctival threading, Müller's muscle, Levator aponeurosis

\section{Introduction}

Along with the increase in the elderly population, the number of patients with age-related ptosis is also on the rise. Various surgical procedures for treatment of ptosis have been previously designed and reported [1]. The transconjunctival method places little burden on patients compared with transcutaneous ptosis surgery and involves low surgical invasiveness; thus, it is a simple surgical method to perform in elderly patients. In 2006, we devised and presented a method to achieve a shortening effect of the levator palpebrae superioris and tarsal muscles by suturing the Müller's muscle and the levator aponeurosis to the tarsus without incision or excision of the tarsal muscle [2]. Subsequently, we have improved the procedure to increase efficacy and expand the indicated cases. Here the new procedure and its efficacy in elderly patients have been reported.

\section{Subjects and Surgical Techniques}

\section{Subjects}

Age-related ptosis was diagnosed in 17 patients (men:

*Corresponding author: Kosuke Ogasawara, M.D., Ph.D, Medical Corporation, Ogasawara Eye Clinic, 3-10-12, Takamatsu, Morioka, 020-0114, Japan, Tel: +81-19-(662)-3223, Fax: +81-19(662)-7331

Accepted: January 29, 2019

Published online: January 31, 2019

Citation: Ogasawara K (2019) Ptosis Surgery with an Improved Method of Shortening the Müller's Muscle and the Levator Aponeurosis with Transconjunctival Threading. J Ophthalmic Surg 2(1):7-12 

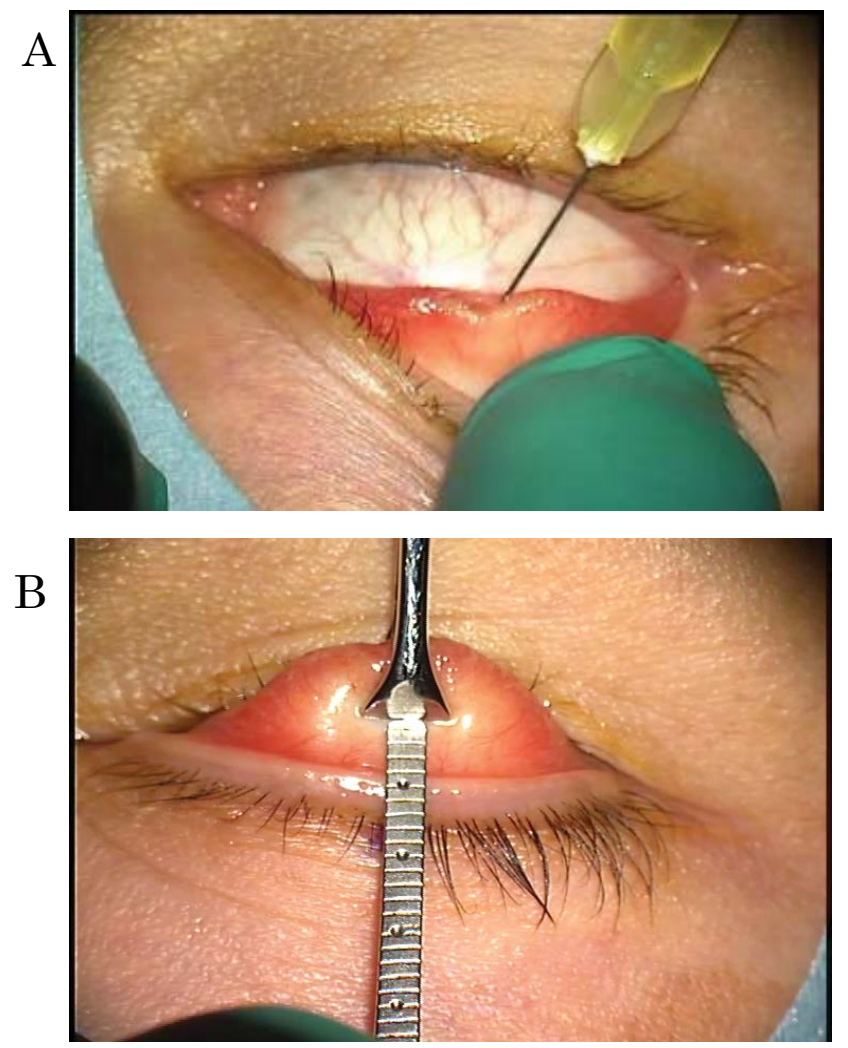

Figure 1: A) A small dose of local anesthesia (1\% lidocaine not containing sympathomimetic drug approximately $0.5 \mathrm{ml}$ ) was administered from the conjunctival fornix; B) The tarsus was pinched by using fixation forceps (INAMI ${ }^{\circledR}$ S-361) so that the tip of the fixation forceps came approximately $5 \mathrm{~mm}$ from the eyelid, and the tarsus was reversed.

7 eyes of 5 patients; women: 22 eyes of 12 patients) with a mean age of $72.3(54-83)$ years. The degree of ptosis in the patients was moderate; the margin reflex distance was $0-3$ $\mathrm{mm}$, and the levator muscle function was $5-8 \mathrm{~mm}$. The results of phenylephrine testing were positive for nine patients and negative for eight patients. Preoperatively, we examined the past medical history of the ptosis patients and confirmed that ptosis was not congenital; neurological examinations showed no brain abnormality, and the condition was not neurogenic.

Written informed consent was obtained from all the subjects. The study was conducted as per the tenets of the Declaration of Helsinki and with the approval of the medical corporation of Ogasawara Eye Clinic's ethical review board.

\section{Surgical techniques}

\section{- $\quad$ Step 1 (Figure 1)}

After ophthalmic anesthesia administration, the upper eyelid was rotated, and a small dose of local anesthesia (1\% lidocaine not containing a sympathomimetic drug; approximately $0.5 \mathrm{~mL}$ ) was administered from the conjunctival fornix. The tarsus was pinched using fixation forceps (INAMI ${ }^{\circledR}$ S-361) so that the tip of the fixation forceps was approximately $5 \mathrm{~mm}$ from the eyelid, and the tarsus was reversed.

- $\quad$ Step 2 (Figure 2)
A

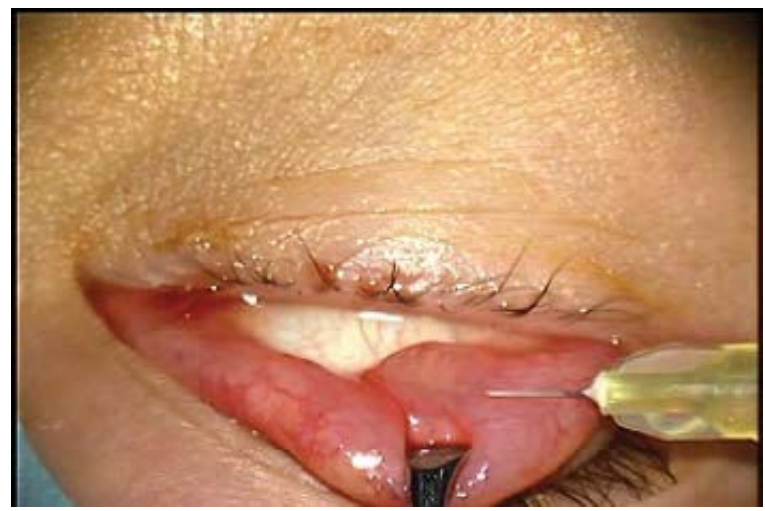

B

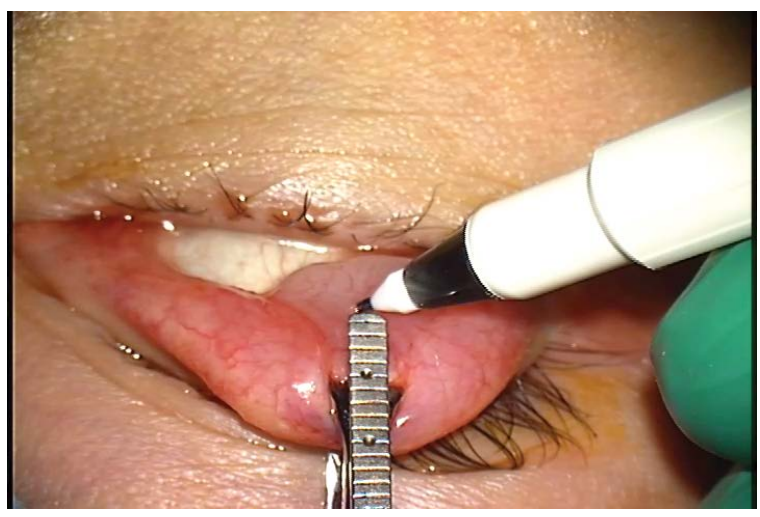

C

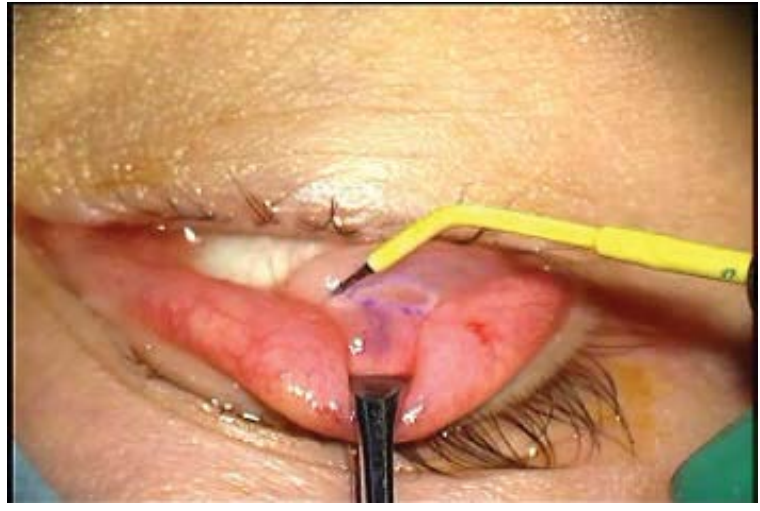

Figure 2: A) A small dose of local anesthesia (1\% lidocaine not containing sympathomimetic drug) was administered with care taken not to damage Müller's muscle; B) Marking incision point at the conjunctiva $5 \mathrm{~mm}$ from the edge of the pinched forceps; C) A high frequency scalpel (ellman ${ }^{\circledR}$ Surgitoron DualEMC90) was used to make an incision as marking line approximately $5 \mathrm{~mm}$ in the conjunctiva.

A small dose of local anesthesia (1\% lidocaine) was administered at a site approximately $5 \mathrm{~mm}$ from the tip of the pinched forceps, with care taken not to damage the Müller's muscle. Thereafter, an approximately 5-mm wide incision was made in the conjunctiva using a high-frequency scalpel (ellman ${ }^{\circledR}$ Surgitoron DualEMC90). Use of scissors is acceptable for making the incision in the conjunctiva.

\section{- $\quad$ Step 3 (Figure 3)}

A 7-0 nylon thread (MANI ${ }^{\circledR} 3365$, double-armed suture) was passed through the Müller's muscle on the tarsal side (approximately $3 \mathrm{~mm}$ from the eyelid margin) under direct vision. Furthermore, from the tarsal side, the thread was 


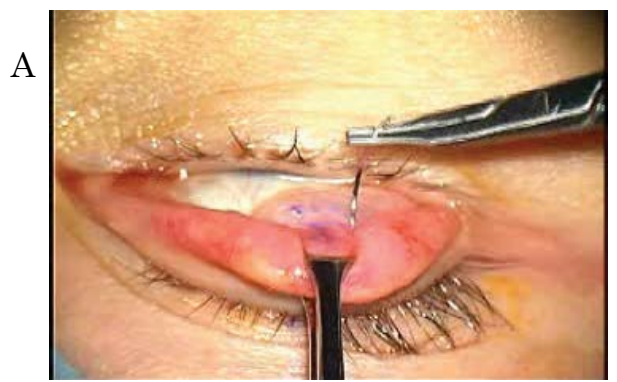

$\mathrm{B}$

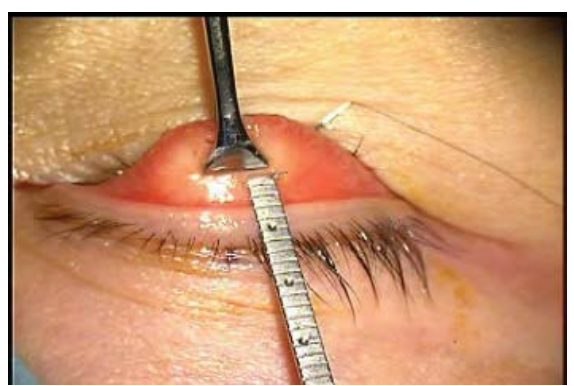

C

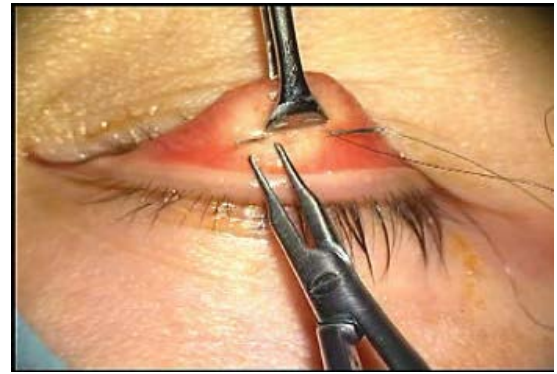

$\mathrm{D}$

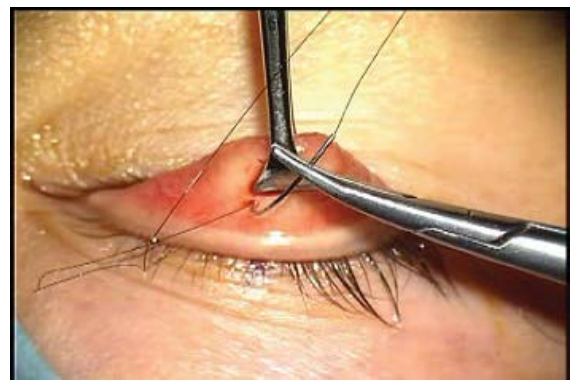

Figure 3: A) A 7-0 nylon thread (MANI ${ }^{\circledR} 3365$, double armed suture) was passed through Müller's muscle and levator aponeurosis on the tarsal side, approximately $3 \mathrm{~mm}$ from the eyelid margin; B) under direct vision; C) From the tarsal side, the thread was passed through the intra-tarsal layer (approximately $5 \mathrm{~mm}$ from the insert point of the needle), and passed it through again on the tarsal side; D) Subsequently rethreading were reformed through the incision in the conjunctival fornix.

A

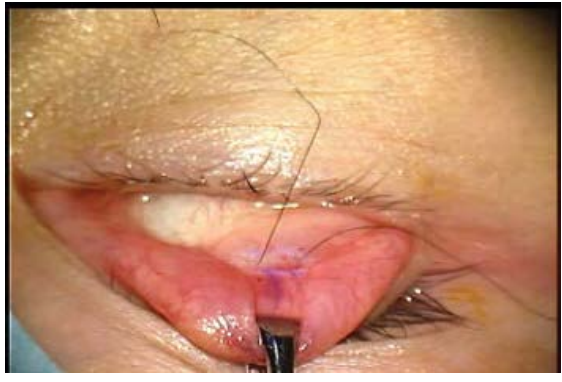

C

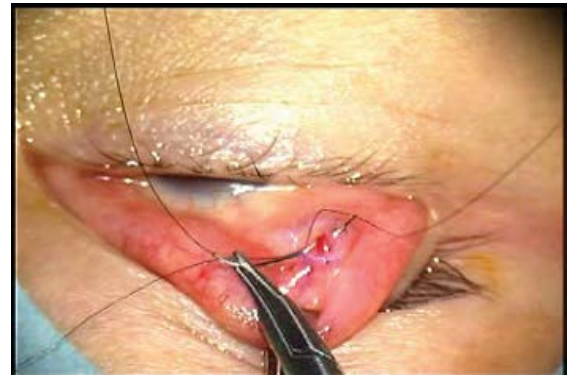

$\mathrm{E}$

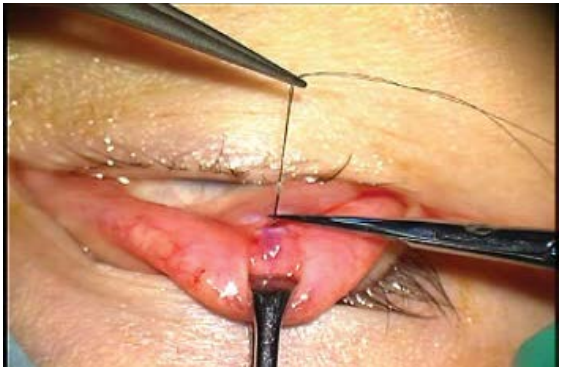

$\mathrm{B}$

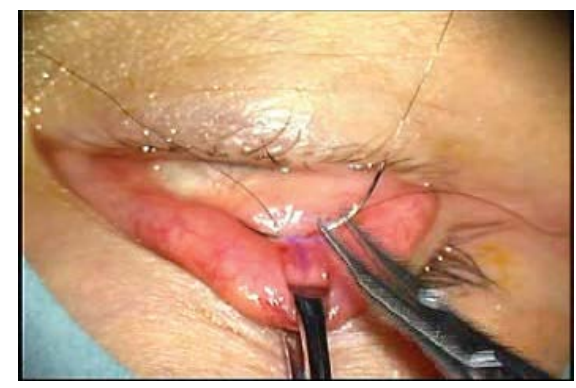

$\mathrm{D}$

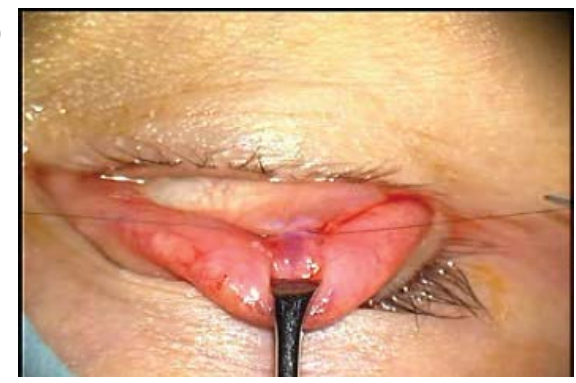

$\mathrm{F}$

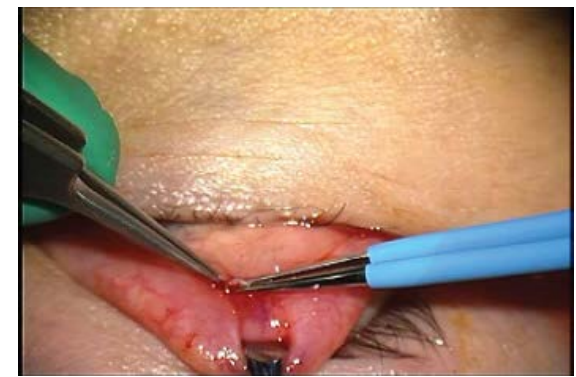

Figure 4: A) Finding after rethreading in the conjunctival fornix; B,C) The each thread was passed from superior to Müller's muscle in a direction traversing the levator aponeurosis; D) Suturing was performed; E) Cutting the suture; F) Bonding of the site of conjunctival incision was performed by coagulation.

passed through the intra-tarsal layer (approximately $5 \mathrm{~mm}$ from the insertion point of the needle) and passed through again on the tarsal side; subsequently, we performed rethreading through the incision in the conjunctival fornix. 


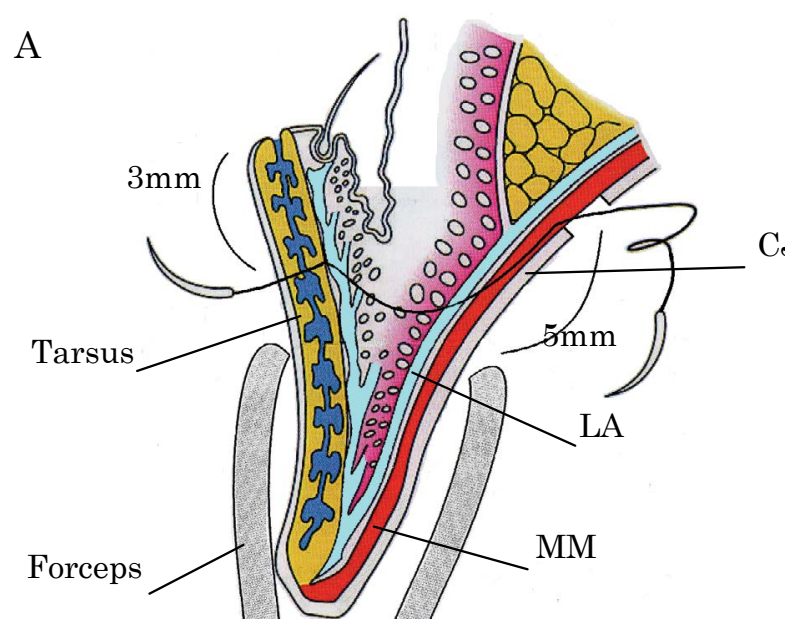

B

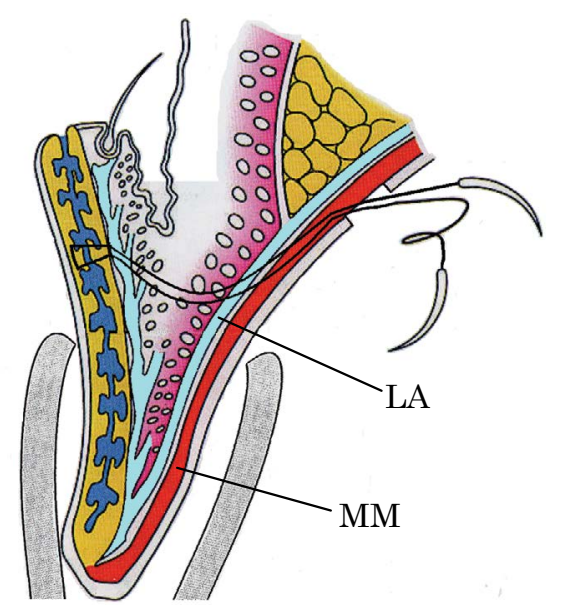

$\mathrm{C}$

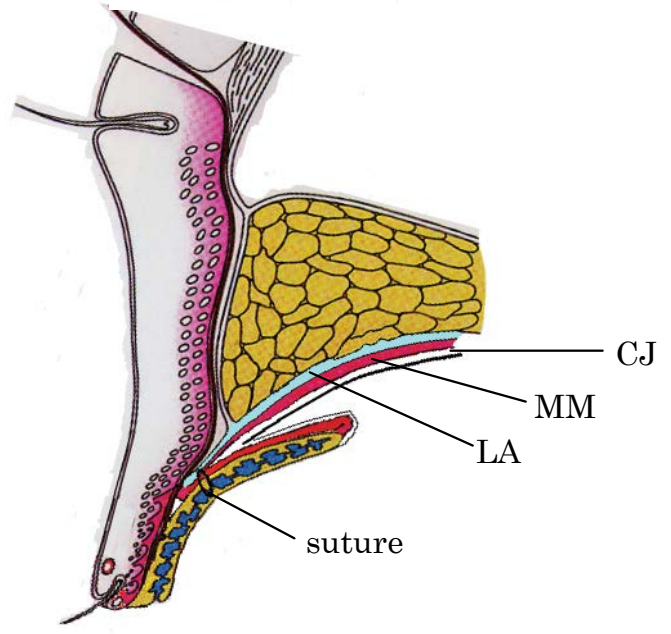

Figure 5: A) After incision in the conjunctiva (CJ) approximately 5 $\mathrm{mm}$ from the edge of the pinched forceps, 7-0 nylon thread was passed through Müller's muscle (MM) and levator aponeurosis (LA) on the tarsal side $3 \mathrm{~mm}$ from the eyelid margin.

B) From the tarsal side, the thread was passed through the intratarsal layer (approximately $5 \mathrm{~mm}$ from the insert point of the needle), and passed it through again on the tarsal side. After then rethreading was performed through the incision in the conjunctival fornix.

C) Finding after finishing the surgery using the present method. Conjunctival incision was bonding by coagulation. Suture was placed in a point superior to Müller's muscle (MM) in a direction traversing the levator aponeurosis (LA).

\section{- $\quad$ Step 4 (Figure 4)}

At that site, the double-armed thread was passed from a point superior to the Müller's muscle in a direction traversing the levator aponeurosis, and suturing was performed. After confirming the eyelid elevation to be good as expected, the suture was then cut and bonding of the site of the conjunctival incision was performed with coagulation. To make symmetry in both unilateral and bilateral ptosis, control of suture tightness was performed in all cases. Mean operative time in this surgery was 11 minutes. A schematic illustration of the present procedures for a sagittal view was showed in Figure 5.

Postoperatively, antibiotic and anti-inflammatory (steroid) ophthalmic eye solutions were administered four times a day for 1 week.

\section{Results}

Follow-up observation was performed for $\geq 3$ months, and satisfactory eyelid elevation effect was achieved in all 17 patients. On postoperative day 1 , mild subcutaneous hemorrhage was observed in one eye, and three patients complained of swelling of the upper eyelid several days postoperatively; however, the swelling disappeared within 1 week following treatment with an ophthalmic solution. There was no case of postoperative development of a corneal disorder. Moreover, there were no cases of postoperative

A

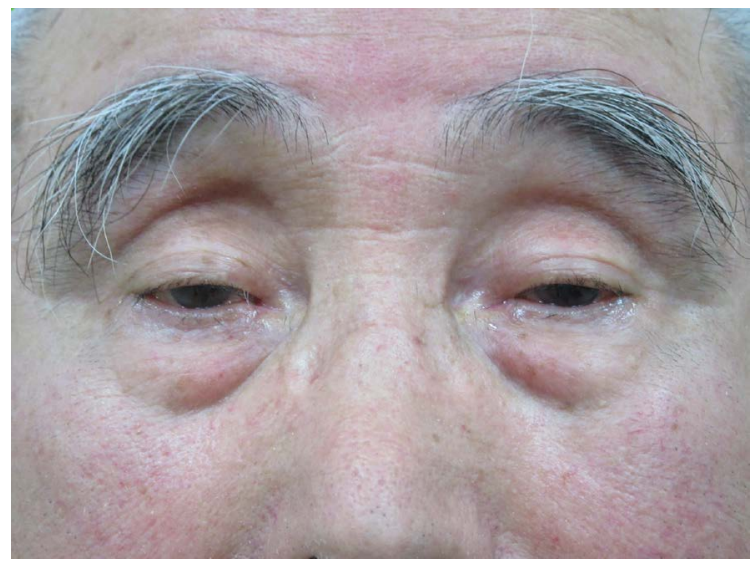

B

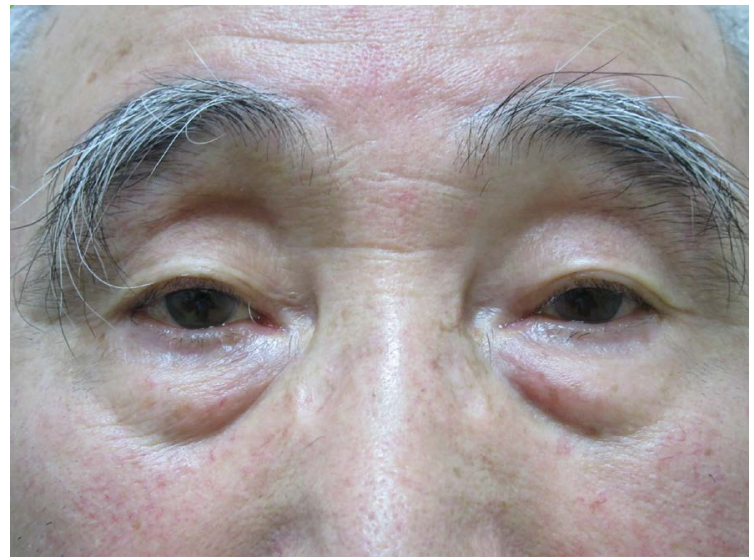

Figure 6: A 82-year-old man with aquired bilateral blepharoptosis with phenylephrine testing negative. A) Preoperative and; B) postoperative three months after surgery. 
entropion or ectropion and incomplete eyelid closure due to overcorrection.

Here we have presented two representative cases among those in whom the procedure was performed (Figure 6 and Figure 7).

\section{Discussion}

The Müller's muscle and levator aponeurosis shortening using transconjunctival threading reported in the present study appear to be effective for common age-related ptosis and may exert the same effect as percutaneous upper eyelid muscle tucking. The reasons for the success of the improved surgical method are as follows: After making a small conjunctival incision, a 7-0 nylon thread was passed through the Müller's muscle from the tarsal side under direct vision, and the threading distance on the tarsal side was approximately $5 \mathrm{~mm}$ inside; moreover, after rethreading into the incision of the conjunctival fornix, the eyelid lift force was increased by threading from a point superior to the Müller's muscle in a direction traversing the levator aponeurosis.

However, this method is not indicated in cases of congenital ptosis or ptosis with complete loss of function of the levator palpebrae superioris.

Although there was a risk for scratching of the cornea due to exposure of the thread at the point of the threading needle during threading on the tarsal surface, the suture was firmly embedded in the tarsus during threading (Figure 8A),
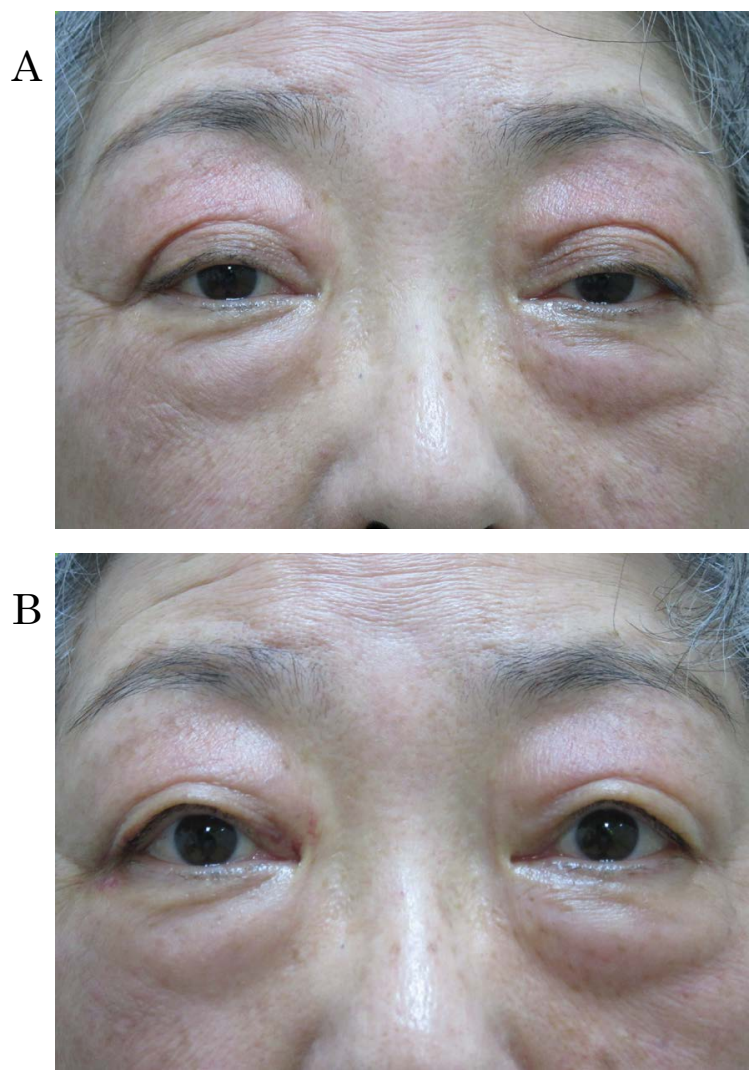

Figure 7: A 71-year-old woman with aquired bilateral blepharoptosis with phenylephrine testing positive. A) Preoperative and; B) Postoperative three months after surgery. and there were no complications. It may also be favorable to slightly widen the point of insertion of the thread needle using the tip of the 27-gauge hypodermic needle (Figure 8B) to prevent thread exposure.

The benefits of transconjunctival ptosis correction surgery include a short surgery time, minimal invasiveness, reduced postoperative pain, fewer postoperative complications, and early social reintegration in elderly patients. Furthermore, simultaneous bilateral surgery of both the eyes is possible. Moreover, the method is advantageous in terms of little impact on the double eyelid fold and no complications such as incomplete eyelid closure due to overcorrection.

The Fasanella-Servat method [3] and the Putterman-Urist method [4] are the most popular transconjunctival methods; however, in both the procedures, the greater degree of surgical invasiveness is a disadvantage. Methods of remedying these drawbacks have also been attempted $[5,6]$ and Shimizu, et al. [6] have reported an excellent method to simultaneously create a double eyelid fold after transconjunctivally burying the nylon thread in the eyelid and performing fixation of the levator aponeurosis to the tarsus. However, this procedure is slightly complex and involves limitations regarding the degree of ptosis and age-related indications. The surgical procedure reported in the present study may be comparable to levator palpebrae superioris muscle shortening in terms of the tucking effect of the Müller's muscle and levator aponeurosis; further, it is possible to control the extent of its effects by changing the threading site after conjunctival incision. Although follow-

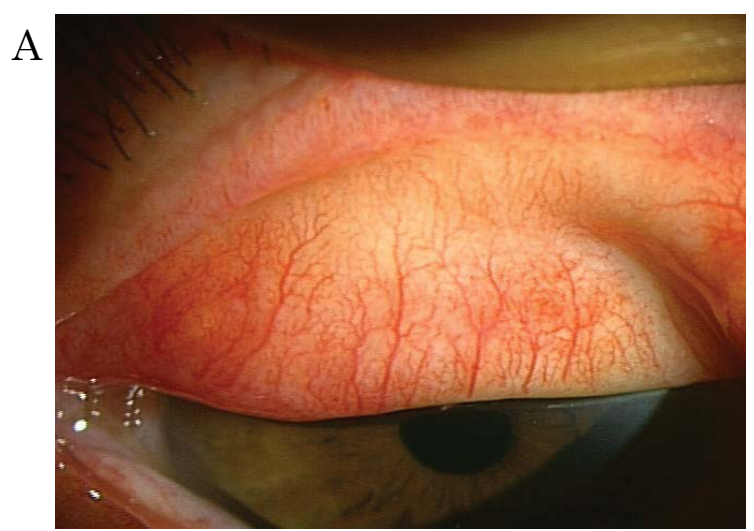

B

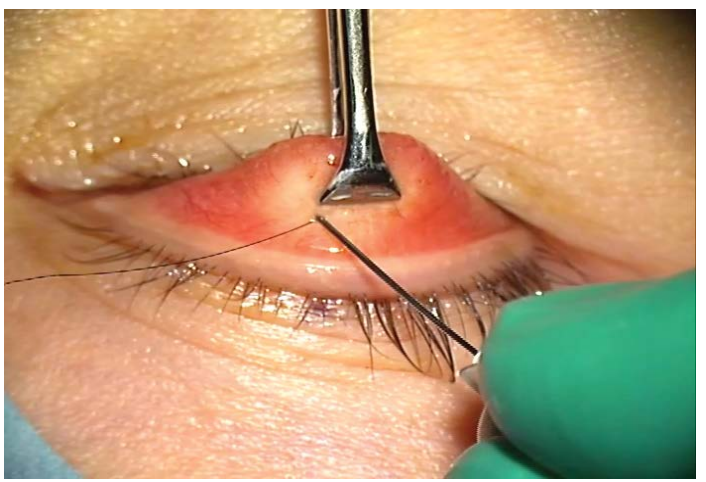

Figure 8: A) The finding showing the thread was firmly embedded in the tarsus one month after surgery; B) Showing widen the insertion point of the threading needle by the tip of the 27-guage hypodermic needle. 

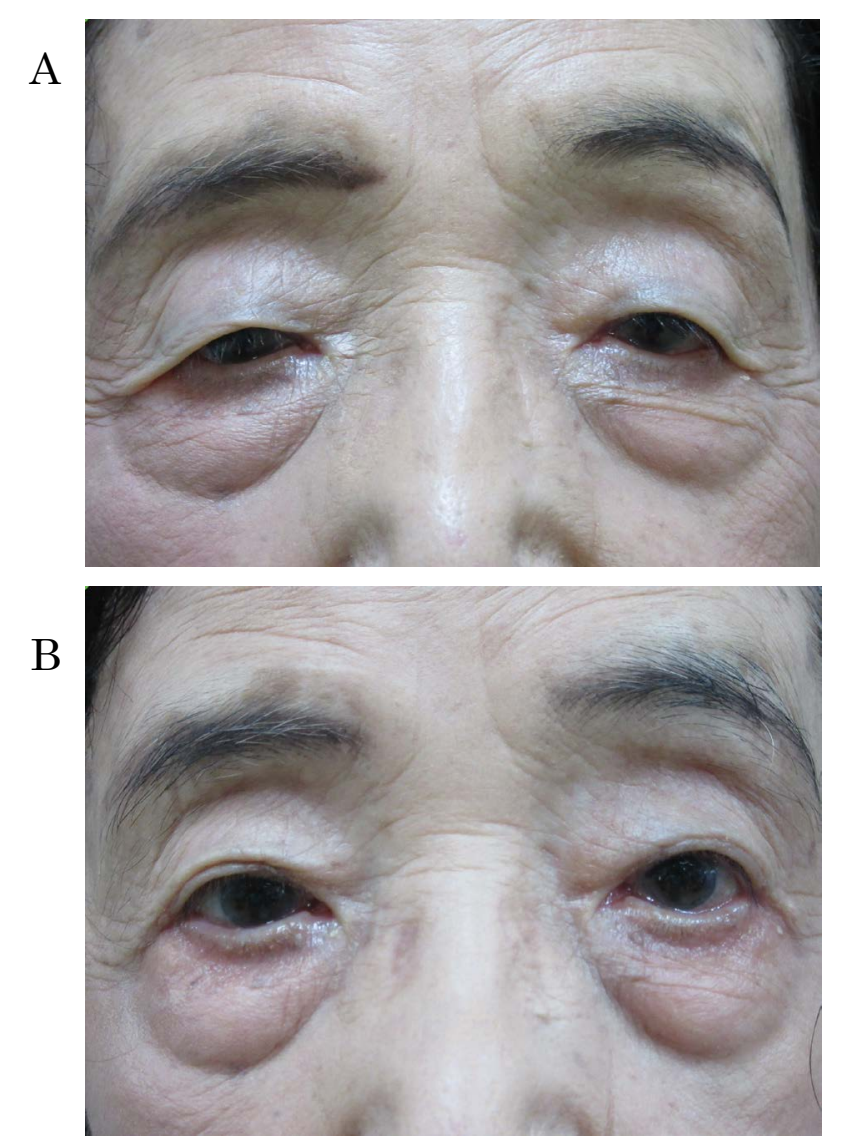

Figure 9: A 87-year-old woman with aquired bilateral blepharoptosis and coexistent blepharochalasis. A) Preoperative and; B) postoperative six months after surgery in which skin resection of the upper eyelid was additionally performed simultaneously.

up observation is necessary for the long-term postoperative course of this method, it is considered superior in terms of its short operative duration; less patient burden such as postoperative pain; and no observations of intraoperative hemorrhage and postoperative overcorrection, entropion, and ectropion. Moreover, in case of patients with coexistent blepharochalasis, skin resection of the upper eyelid can be additionally performed simultaneously (Figure 9), and if the correction effect is insufficient, the method can be converted to another surgical procedure.

The above results suggest that the present procedure may be a favorable method for initial surgery in age-related and adult ptosis. Regarding the mechanism of success of this surgical procedure, in the conventional percutaneous levator palpebrae superioris tucking method, the tarsal muscle is shortened in a folding form, whereas in the present method, the force performing traction from the tendon part of the tarsal muscle works more strongly, thus, enhancing the function of the levator palpebrae superioris muscle; this appears to be the most important factor in the success of this method. Basic physiological [7] and clinical [8] studies have demonstrated that the Müller's muscle functions as a stretch receptor and is the primary inclusion that transmits the contraction of the levator palpebrae superioris muscle to the tarsal plate. Furthermore, contraction of the Müller's muscle transmits afferent input to the mesencephalic nucleus of the trigeminal nerve [7]. Therefore, it is suggested that in the present method, due to the traction of the levator aponeurosis, the levator palpebrae superioris muscle itself may be rotated anteriorly to obtain elevation of the upper eyelid, and the tension of the Müller's muscle causes a proprioception effect on the levator palpebrae superioris muscle. Several studies $[9,10]$ have demonstrated that the surgical effect of shortening the Müller's muscle can be achieved in phenylephrine test-negative patients as well similar to that observed in phenylephrine test-positive patients. These findings are supported by the efficacy of the surgical method observed in the present study and its mechanism of success.

\section{Disclosure}

The author reports no conflicts of interest in this work.

\section{References}

1. Anthony Tyers, JRO Collin (2008) Color atlas of ophthalmic plastic surgery. Elsevier, Japan, 170-213.

2. Odashima S, Ogasawara K (2006) New suture technique to correct ptosis without incision of the eyelid or the conjunctiva. Jap J Ophthalmic Surg 19: 579-582.

3. Fasanella RM, Servat J (1961) Levator resection for minimal ptosis: Another simplified operation. Arch Ophthalmol 65: 493496.

4. Putterman AM, Urist MJ (1975) Müller muscle-conjunctiva resection. Technique for treatment of blepharoptosis. Arch Ophtalmol 93: 619-623.

5. Ichinose A, Tahara S (2007) Transconjunctival levator aponeurotic repair without resection of Müller's muscle. Aesthetic Plast Surg 31: 279-284.

6. Shimizu Y, Nagasao T, Asou T (2010) A new non-incisional correction method for blepharoptosis. J Plast Reconstr Aesthet Surg 63: 2004-2012.

7. Matsuo K (2002) Stretching of the Mueller muscle results in involuntary contraction of the levator muscle. Ophthalmic Plast Reconstr Surg 18: 5-10.

8. Fujiwara T, Matsuo K, Kondoh S, et al. (2001) Etiology and pathogenesis of aponeurotic blepharoptosis. Ann Plast Surg 46: 29-35.

9. Baldwin HC, Bhagey J, Khooshabeh R (2005) Open sky Müller muscle-conjunctival resection in phenylephrine test-negative blepharoptosis patients. Ophthalmic Plast Reconstr Surg 21: 276-280.

10. Rootman DB, Karlin J, Moore G, et al. (2015) The role of tissue resection length in the determination of post-operative eyelid position for Muller's muscle-conjunctival resection surgery. Orbit 34: 92-98.

DOI: $10.36959 / 587 / 589$ 